\title{
First-pass myocardial perfusion imaging with whole ventricular coverage using L1-SPIRIT accelerated spiral trajectories
}

\author{
Yang Yang ${ }^{1 *}$, Xue Feng ${ }^{1}$, Craig H Meyer ${ }^{1,2}$, Christopher M Kramer ${ }^{3,2}$, Michael Salerno ${ }^{3,2}$ \\ From 16th Annual SCMR Scientific Sessions \\ San Francisco, CA, USA. 31 January - 3 February 2013
}

\section{Background}

First-pass perfusion imaging using cardiac magnetic resonance (CMR) has become clinically applicable as an important tool for diagnosing coronary artery disease. We have recently demonstrated that high quality firstpass images can be acquired with an optimized spiral pulse sequence, but this sequence is only capable of imaging 3 short axis slices of the heart for a maximum heart rate of $110 \mathrm{bpm}$. In order to achieve whole ventricular coverage, we have developed an accelerated spiral sequence using the Parallel Imaging and Compressed Sensing technique L1-SPIRIT.

\section{Methods}

To evaluate and develop the accelerated reconstruction we retrospectively down-sampled data acquired from a fullysampled variable density (VD) spiral perfusion data set acquired on a $1.5 \mathrm{~T}$ scanner. Pulse sequence parameters included: TE $1.0 \mathrm{~ms}$, TR $11 \mathrm{~ms}$, FOV $320 \mathrm{~mm}^{2}$, in-plane resolution $1.75 \mathrm{~mm}, 8$ interleaves, readout duration $8.1 \mathrm{~ms}$ per interleave, saturation time $8 \mathrm{~ms}$. We then designed a $4 \mathrm{x}$ accelerated pulse sequence which prospectively collected only 2 of 8 interleaves, and collects two perfusion images after each saturation pulse. Resting perfusion imaging was performed after injection of $0.1 \mathrm{mmol} / \mathrm{kg}$ of

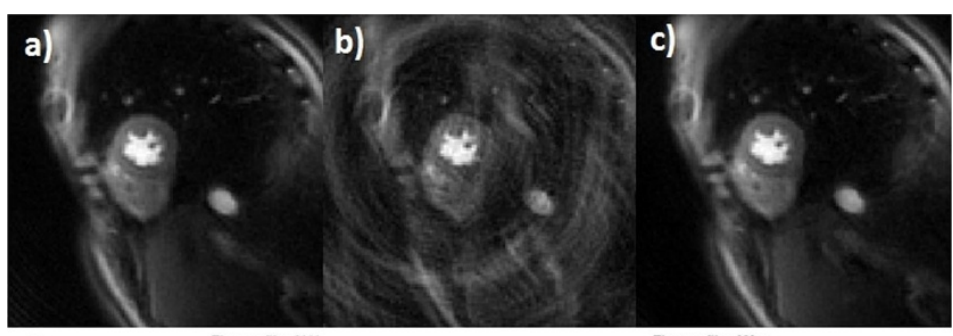

d)

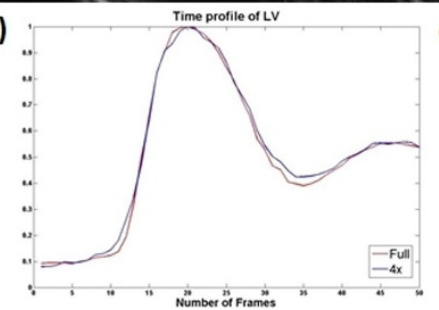

e)

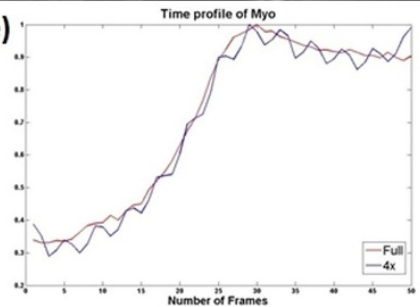

Figure 1 Retrospective perfusion images with fully sampled(a), 4x direct reconstruction with zero-pad (b) and the I1-SPIRiT recon (c), and temporal profiles of fully sample (red) and I1-SPIRiT (blue) from LV cavity (d) and myocardium (e)

'Biomedical Engineering, University of Virginia, Charlottesville, VA, USA

Full list of author information is available at the end of the article

(C) 2013 Yang et al; licensee BioMed Central Ltd. This is an Open Access article distributed under the terms of the Creative Commons 


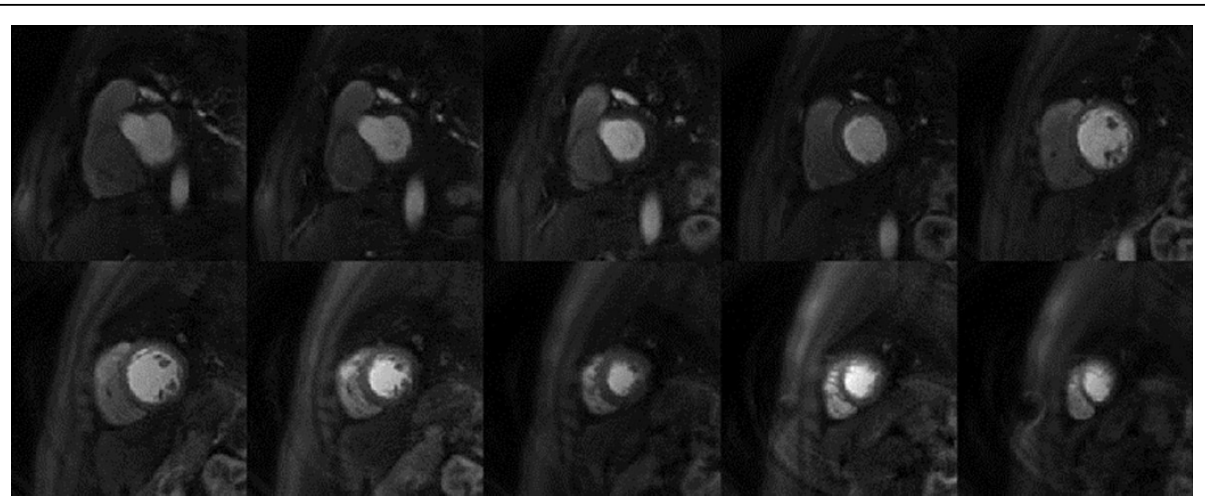

Figure 2 Perfusion images of 10 slices covering the whole ventricular myocardium

Gd-DTPA. Proton-density images acquired at the beginning of the acquisition were used to train the SPIRIT calibration kernel. Data reconstruction was performed using an iterative conjugate gradient reconstruction including a data fidelity term, SPIRIT calibration consistency term and an L1-finite difference in time as the sparsifying transform. Reconstruction was implemented in Matlab.

\section{Results}

Figure 1 shows the fully-sampled image (a), 4x direct reconstruction with zero-pad (b) and the L1-SPIRiT accelerated results from the retrospective perfusion data. At an acceleration factor of 4, this L1-SPIRIT reconstruction resulted in excellent image quality. Temporal profiles from the LV cavity (d) and myocardium (e) were generated to assess the temporal fidelity of this reconstruction. The L1-SPIRIT curves (blue) are almost identical to the fully sampled (red) curves. The temporal oscillation on tissue function curve for the $4 \mathrm{x}$ accelerated images is resulting from the down sampling pattern and does not occur with actual data acquisition. Figure 2 shows perfusion images acquired with the $4 \mathrm{x}$ accelerated L1-SPIRIT pulse sequence which enables acquisition of 10 slices covering the whole ventricular myocardium.

\section{Conclusions}

We demonstrated the successful application of whole ventricular coverage first-pass myocardial perfusion imaging using accelerated spirals. This sequence can acquire 8 short axis slices in $480 \mathrm{~ms}$ enabling full ventricular coverage at heart rates up to $125 \mathrm{BPM}$. Further validation will be required in patients undergoing adenosine stress CMR.

\section{Funding}

This work was supported by grants from AHA 10SDG2 650038 and NIH K23 HL112910-01.

\section{Author details}

'Biomedical Engineering, University of Virginia, Charlottesville, VA, USA. ${ }^{2}$ Radiology, University of Virginia, Charlottesville, VA, USA. ${ }^{3}$ Medicine, University of Virginia, Charlottesville, VA, USA.

Published: 30 January 2013

doi:10.1186/1532-429X-15-S1-P20

Cite this article as: Yang et al:: First-pass myocardial perfusion imaging with whole ventricular coverage using L1-SPIRIT accelerated spiral trajectories. Journal of Cardiovascular Magnetic Resonance 2013 15(Suppl 1):P20.

\author{
Submit your next manuscript to BioMed Central \\ and take full advantage of: \\ - Convenient online submission \\ - Thorough peer review \\ - No space constraints or color figure charges \\ - Immediate publication on acceptance \\ - Inclusion in PubMed, CAS, Scopus and Google Scholar \\ - Research which is freely available for redistribution \\ Submit your manuscript at \\ www.biomedcentral.com/submit
}

